\title{
Representation of innovations in the model of regional development
}

\author{
Vladimir Gurman \\ Program Systems Institute RAS \\ 152140 Pereslavl, Russia \\ E-mail gurman@cprc.botik.ru \\ Elena Ryumina \\ Market Economy Institute RAS \\ Nakhimovsky pr.47, Moscow, 117418, Russia \\ E-mail: ryum@ccas.ru
}

\section{Introduction}

An important factor of regional development is innovative process. The innovations include new products, new technologies, new ways of commercial use of goods conquering new markets, new sources of raw materials and other qualitative trahsformations which can change current economic situation. Thus the innovations can be considered as an instrument for icreasing of economic effectivity leading to the market success. Of course they were stimulated by strong competition of companies, and now become the basic buisness strategies in which knowledge and social capital create the competitive advatages of regions more than their natural resources. 
Morover, the knowledge are going out of inner production cicles and to be an independent product creating a new economic sector; thus one can speak about growing of innovative economy as a new type of economy (Konstantinov, 1999).

One can consider such a course as positive from the point of view of sustainable development paradigm which is characterized by requirements of more and more restricted use of Nature in the traditional sence, and of creation the natural resources and environment (natural capital) reproduction cicles which along with social capital should provide the living and development conditions for the future generations. Such requirements can be fulfilled only by intensive use of sceintific/technological achievements, that is via high innovative activity. At the same time innovations may have negative ecological effect, and such processes as technological improvement may be accompanied by such negative sical phenomena as unemploiment. This means that when developing innovative strategies one should weight their "pluses" and "minuses" in all set of their effects, and that in mathematical models that are intended to describe satisfactory the sustainable development the innovative processes should be presented properly. The main methodological problem is that these processes are incorporated into real picture of development whereas it is necessary to separate them from other processes in the integrated model to perform their fruitful analysis, in particular to compare the related costs with the total resulted effect. Our approach to construct the model with innovations is to modify a familiar previously created model without innovations via addition a special submodel which describe visually the innovative processes, and to understand under the term "innovations" any favorable change of the original model parameters (including prices for products due to their improvement/renovation). This can be interpreted as increasing of the regional competability under sustainable development requirements. The output of this submodel is the changing set of the original model parameters depending on intensity of innovative processes, and its in- 
put includes a series of the original variables such as social quality index, investment and import flows, which influence on the innovative activity. The main difficulty arise when we attempt to present strictly the dynamics of great amount of the original model parameters (about square of the number of original variables) which results in much more greater amount of new parameters (about 4-th degree of the number of original parameters). To overcome this difficulty the opportunity of aggregative description of innovative block with different levels of aggregation up to one state variable is provided. Also new requirements for empirical base of such submodel and for the overall model arise. The modified model structure become essentially nonlinear and does not allow to apply strictly the special high effective approximate optimization method used earlier to ivestigate the original model. For this reason a multistep optimization procedure is developed where optimization of the original model with given parameters is accompanied by their inprovement on account of control in the innovative submodel

\section{Brief description of the innovative sub- model}

In the model, the innovations are expressed by changes in the values of the parameters of the original submodel described conventionally by the following general vector relations

$$
\dot{x}=f(t, x, u, a), \quad(x, u) \in \mathbf{B}(\mathbf{a}),
$$

where $x$ and $u$ are the vectors of original state and control variables, and $a$ is the vector of original parameters.

The number of parameters can be very large: roughly speaking, it is proportional to the squared number of state variables in the model. We shall therefore in practice not work with the vector $a=\left(a_{i}\right)$, but with a lower-dimensional, aggregated vector $\theta$. This vector is 
obtained with the help of the following procedure. We first partition the elements of the vector $a$ in $m$ subsets $I_{j}, j=1,2, \ldots, m$. To each subset $I_{j}$ corresponds one element $\theta_{j}$ of $\theta$. The current value of $\theta_{j}$ is constructed according to:

$$
\theta_{j}=\left(\sum_{i \in I_{j}} \frac{\left|\Delta x_{i}\right|}{\left|x_{i}\right|}\right) \frac{1}{n_{i}}
$$

where $a_{i}$ is the current value of the $i$-th parameter in $I_{j} ; n_{j}$ is the number of parameters in $I_{j} ; \Delta x_{i}=x_{i}-a_{i}(0)$; and $x_{i}(0)$ is the initial value of $a_{i}$. Thus, $\theta_{j}$ describes the mean percentage change for the subset of parameters $I_{j}$.

The values of $\theta_{j}$ are disaggregated when required by "redistributing" them for each $t$ among the parameters belonging to the subset $\mathbf{I}_{\mathbf{j}}$. Several rules can be used to perform this disaggregation, in particular:

- by changing all parameters in the subset by the same percentage;

- by weighting the changes according to the level of saturation of each given parameter, that is, its distance from its possible value boundary;

- according to empirical statistical distributions for the parameters;

- using an additional conditional optimization with respect to the parameters subject to $\theta_{j}$ - this last approach allowing to better approximate the true optimum of the original, disaggregated problem.

For this purpose we use the following formula

$$
a_{i j}=\left(1+\theta_{j} \alpha_{i j}\right), \quad i \in \mathbf{I}_{\mathbf{j}}
$$

where $\alpha_{i j}$ are are distributive weight parameters which can be chosen differently according above different distributing rules. 
The model with innovations for a region is constructed as a modification of the general model described in (Gurman, 1981)

$$
\begin{gathered}
c=(E-A) y-B u-A^{z} z-B^{z} w \\
0 \leq y \leq \Gamma(k), \quad 0 \leq z \leq \Gamma^{z}\left(k^{z}\right) \\
\dot{r}=\frac{d r^{*}}{d t}+N\left(r-r^{*}\right)-C y+C^{z} z+i m^{r}-e x^{r} \\
\dot{k}=u-\delta k, \quad \dot{k}^{z}=w-\delta^{z} k^{z} .
\end{gathered}
$$

Here $y$ is current traditional output; $z$ is output vector of the environmental sector; $u$ and $w$ are investments in traditional capital $k$ and in capital $k^{z}$ of the environmental sector; $c$ the real consumption; $r$ is a vector describing the state of the environmental sphere. $E$ is the identity matrix and $A, B, C, D, A^{z}, B^{z}, C^{z}$, and $D^{z}$ are coefficient matrices of appropriate dimensions; $\delta$ and $\delta^{z}$ are diagonal matrices of depreciation rates

The innovative part is described by

$$
\begin{gathered}
\dot{\theta}=-\left([d]+H_{\mathrm{inv}}+H_{\mathrm{diff}}\right)(\theta-\bar{\theta}), \quad \theta(0)=0, \\
\dot{k^{d}}=u^{d}-\delta^{d} \times k^{d}, \quad 0 \leq d \leq \Gamma^{d}\left(k^{d}\right),
\end{gathered}
$$

where $d$ is a control vector representing the activity of innovative sector which capital, capacity and depreciation rate are correspondingly $k^{d}, \Gamma^{d} k^{d}$, and $\delta^{d},[d]$ is a diagonal matrix as "diagonalized" vector $d$, matrices $H_{\text {inv }},[d]$, and $H_{\text {diff }}$ correspond to three main sources of innovations:

1)the investment which typically imply replacing older vintage capital with newer one, with better characteristics;

2)specific expenditures for technology and management improvement that have no direct capacity effect;

3)innovative diffusion processes and similar endogenous innovative changes. 
Typically, $\bar{\theta}$ may represent the best available innovative level in similar regions.

Matrices $[d], H_{\text {inv }}, H_{\text {diff }}$ are assumed nonnegative, which means nonworsening of any aggregated indeces when others are improved. For the original parameters it is not necessary. By choosing some $\alpha_{i j}$ negative in (2) one can represent the effects of improvement a part of parameters on account of worsening some others, and to solve the problems of optimal choice among different versions of such innovations.

The positive impact of the basic capital, socium and environment quality can be accounted for via multiplying the right-hand side of (2) by some matrix $K$ depending on related state variables.

The parameter vector $a$ in (2) is defined as the vector of all stacked elements of the model's coefficient matrices, i.e. of the coeficient matrices and other parameters or their inverses so that "innovative improvement" means reducing the components of $a$.

As a result a complex nonlinear model is obtained even if the original model is linear

\section{The optimization problem}

The following intertemporal welfare function is assumed

$$
J_{T}=\int_{0}^{T}\left[(1-\mu) V-\mu W\left(t, k, k^{z}, r\right)-u^{d}-Q^{d}\right] e^{-\rho t} d t
$$

where $\rho \geq 0$ is the discount factor, $T<\infty$, and $\mu \in[0,1], V=p c$, $u^{d}$ is the vector of investments in $k_{d}, Q_{d} d$ is the current innovative cost, and $W\left(k, k^{z}, r\right)$ is a penalty function for the violation of "soft" sustainability constraints,

$$
\left(k, k^{z}, r\right) \in \mathbf{\Psi}_{\mathbf{s}}(\mathbf{t}),
$$

Formally (6) is represented as

$\dot{J}=\left[(1-\mu) V-\mu W\left(t, k, k^{z}, r\right)-u^{d}-Q^{d}\right] e^{-\rho t}, \quad J(a)=0, \quad J(T)=J_{T}()$ 
The problem of inteest is to find a time profile for the control vectors $y, u, z, w, d$ and $u^{d}$, that maximizes (6) subject to the model relations, the additional constraints on controls

$$
(y, u, z, w, d) \in \Omega\left(t, k, k^{z}, r\right),
$$

"hard" sustainability constraints

$$
\left(k, r, k^{z}\right) \in \Psi_{h}(t),
$$

and given initial and terminal boundary conditions for $k, r, k^{z}, \theta$, and $k^{d}$.

We do not attempt to formulate above constraints more precisely in the general case - its specification will depend on the concrete case considered. Alternatively, other type of terminal constraints are possible.

The complexity and nonlinearity of the resulting integrated model does not allow one to use for it directly any special optimization method. However, if the original model does allow to do it in analytical way, may be approximately, then this opportunity can be used via the following multistep procedure.

Step 1. Disregarding the equation $\dot{\theta}$, the different model's coefficients are considered as arbitrary given functions of time.

Step 2. The thus defined optimal control problem is solved by the special method as mentioned above undera series of idealized assumptions.

Step 3. The control trajectory obtained under Step 2 is locally improved on account of control program $\{d(t)\}$.

Step 4. Steps 1-3 are repeated until no improvement is possible.

Step 5. The idealized assumptions are removed, and the solution is modified correspondingly by an iterative improvement method (see Belyshev and Shevchuk, 1999)

In the steps $1-2$ we assume the following:

1) the controls $u$ and $z$ are unbounded; the equation for $\dot{k}^{z}$ in(??), is dropped, and the term $B_{z} w$ in (2) is taken zero; 
2) the boundary conditions on $k, k^{z}$, and $r$ are fix point ones.

To solve these steps, we base on the extension principle (Gurman $1985,1997)$ which idea is to replace the original problem by an analogous one but free of some "difficult" constraints so that its solution either exactly coincides with or lies close to the solution of the original problem. Specifically we use "singular relaxation" method where the extensions considered are so called singular relaxations, i.e. extensions that, applied to a class of unbounded = control systems, preserve their all integral characteristics. They allow to represent generalized optimal solutions as impulse modes in terms of regular ordinary differential equations.

According to the "singular relaxations" method we find the integral:

$$
I=J+(1-\mu) \Lambda_{k} k+\Lambda_{r} r
$$

where

$$
\Lambda=(1-\mu) \tilde{p}\left(B+A^{z}\left(C^{z}\right)^{-1} D\right), \quad \Lambda_{r}=(1-\mu) \tilde{p} A^{z}\left(C^{z}\right)^{-1},
$$

for the following auxiliary system (basis system):

$$
\begin{array}{ll}
\frac{\partial k}{\partial \tau_{1}}=E ; & \frac{\partial r}{\partial \tau_{1}}=-D ; \quad \frac{\partial J}{\partial \tau_{1}}=-(1-\mu) \tilde{p} B ; \\
\frac{\partial k}{\partial \tau_{2}}=0 ; & \frac{\partial r}{\partial \tau_{2}}=C^{z} ; \quad \frac{\partial J}{\partial \tau_{2}}=-(1-\mu) \tilde{p} A^{z}
\end{array}
$$

where $\tau_{1}, \tau_{2}$ are multidimensional auxiliary arguments, $\tilde{p}=e^{-\rho t} p$.

We then compute the full derivative of (8) using the system(4),(5), and (3), to obtain

$$
\begin{aligned}
\dot{I}= & (1-\mu) \tilde{p}\left(E-A-A^{z}\left(C^{z}\right)^{-1} C\right) y-\mu W\left(t, k, k_{z} r\right)+\dot{r}^{*}+ \\
& +\left(\dot{\Lambda}-\Lambda \delta k+\left(\dot{\Lambda}_{r}+\Lambda_{r} N\right) r+\right. \\
& +\Lambda_{r}\left(i m_{r}-e x_{r}-N r^{*}+\dot{r}^{*}\right),
\end{aligned}
$$

where

$$
\Lambda=(1-\mu) \tilde{p}\left(B+A^{z}\left(C^{z}\right)^{-1} D\right), \quad \Lambda_{r}=(1-\mu) \tilde{p} A^{z}\left(C^{z}\right)^{-1} .
$$


This is a first-order control system which does not depend upon the original control variables. Any regular solution of the original system is a solution of this new system. The converse, however, is not true.

Taking into account equation (??) and the boundary conditions on $k, k^{z}$, and $r$, it is clear that the minimum of $J(T)$ corresponds to the minimum of $I(T)$, that in turn is achieved by minimization of the right-hand side of (11) with respect to $y, k$, and $r$, subject to the given constraints.

Let

$$
\kappa=p\left(E-A-A^{z}\left(C^{z}\right)^{-1} C\right) .
$$

It is a row-vector with components $\kappa_{i}, i=1,2, \ldots$. Then $y_{i}=\Gamma_{i}$ if $\kappa_{i}>0, y_{i}=0$ if $\kappa_{i}<0$, and $y_{i}$ is arbitrary if $\kappa_{i}=0$. These values of $y_{i}$ are substituted into the right-hand side of (11) and the resulting function is minimized with respect to $k$ and $r$.

The result is a set of functions $\{\hat{y}(t), \hat{k}(t), \hat{r}(t)\}$, called turnpike. The combination of a turnpike with the given boundary values is as a rule discontinuous. We can approximate this turnpike with any given accuracy by a sequence of functions: with infinitely increasing time derivatives in the vicinity of each discontinuety pint. This being done, we put this sequence into the equations (5) and (4), and resolve these equations with respect to the control variables to obtain optimal control sequences $u_{q}(t)$ and $z_{q}(t)$.

Thus, we obtain the generalized solution of Step 2. This solution is completely characterized by the turnpike found almost analytically from the finite-dimensional minimization of the right-hand-side of $\dot{I}$.

At Step 3 we consider the minimization of $I(T)$ taking into account the (11) and the equation (2) omitted earlier. We substitute the turnpikes obtained at step 3 into the right-hand side of (11) and express all matrices as a functions of $\theta$ so that (11) is transformed into an equation of the form:

$$
\dot{I}=\Phi(t, \theta, d)
$$


Then we make a one-step local improvement of $d(t)$ for the system (12) and (2), given $I(0)=I_{0}, \theta(0)=0$. We then return to steps 1 and 2 .

An important result of the step 2 of the optimization procedure for the model under consideration is a criterion of regional structural efficiency under sustainable development requirements $\kappa(3)$ which sence is simulteneous generalized productivity of the aggregated branches of economy that is all branches optimal outputs should be maximal, otherwise some of them should be minimal.

\section{Application to Pereslavl region}

Pereslavl region is a part of Yaroslavl oblast of Russia. Its center, Pereslavl-Zalessky town, is situated on the famous Golden Ring of Russia, halfway from Moscow to Yaroslavl city, on the beach of Plestcheevo lake (aquatory of 51 sq.kilometers)which has the status of a natural and historical memorial. Its territory is of 3300 sq.kilometers, and present population is about 71 thousands.

The leading branches of economy are photochemical, textile, food industries, and agriculture. There is a great potential for turism and recreation taking into account comparatively good ecological conditions and numerous beautiful landscapes, historical relics and monuments.

The innovation structure in Pereslavl region is mainly defined by its industrial structure. The main purpose of innovations in leading industries is to provide the competitiveness of enterprises. The list of new products have been increasing by 100 percents for last 3 years.

For example, LIT, former photochemical factory, began to produce new high effectife heat-reflecting construction films and panels with heat saving up to $30-40$ percents.

An important direction is new technologies oriented to natural resorces saving (about 20 percents of total investment). In particular, high effectife technology and facility for catalitic gas cleaning 
have been developed in LIT company providing $97 \%$-cleaning of atmospheric discharges and at the same time saving about $70 \%$ of outgoing heat.

A serious obstacle for innovative investments flow is due to bad state of industrial and social infrastructure: roads, communications, water and gas supply, buildings and facilities for scools, hospitals, hotels, etc.

In order to help the increasing the innovative activity, in 1995 it was opened Pereslavl university, the first one in a small town of Russia, by initiative of local scientific community, municipal administrations and leading enterprices. Also a regional Innovative Center is considered to create on the basis of the Program Systems Institute of Russian Academy of Sciences (PSI RAS)

All this tells in favor of modeling and investigation in multivariant computer experiments the optimal sustainable development strategy of the region in order to use most effectively the development potential awailable. To build the concrete regional model on the basis of the above abstract one it is necessary to define all state variables and to specify quantitavely all parameters and unspecified functions with the help of the regional statistics and additional empirical research.

The economic activities of the region have been aggregated into three branches:

- 1 represented by those activities which are highly specific to Pereslavl, namely photochemistry, films and textile;

- 2 including the most traditional sectors (e.g. agriculture, building, transport, communications, etc.);

- 3 which is primarily referring to the non-productive, social services sector (education, financial benefits, etc.).

The capitals $k_{i}$ and outputs $y_{i}, i=1,2,3$ of these branches are measured in millions roubles with constant prices as prices at the end of 1995. 
Vector $r$ was assumed consisting of four components $\left(r_{1}, r_{2}, r_{3}\right.$, $\left.r_{4}\right)$. The first one $\left(r_{1}\right)$ is Gross Stock of Natural Resources Index (NRI), representing the sum of supplies of the natural resources (in this case peat, sand, clay, timber, fish, game, and berries), measured in millions of roubles given conventional prices as prices of the primery raw materials produced of these recourses.

The second one $\left(r_{2}\right)$ is Natural Quality Index (NQI), a weighed sum of concentrations of the main water and air pollutants (SS, BOD, oil, nitrogen, copper, zinc, and dust, SO2, CO, NOx, CHx respectively) each normalised (divided) by its Maximum Allowable Concentration (MAC).

The third one $\left(r_{1}\right)$ is population of the region (thousands), and the forth one $\left(r_{4}\right)$ Social Development Index (SDI) which was chosen to follow a procedure similar enough to the Human Development Index (HDR, 1995) with the use of normative aspects of many of the indicators:

- health, characterised by average duration of life;

- material well-being, including per capita floor space, durable goods, income per capita, and financial benefits;

- culture and education, formed by proportion of people with higher degree, participation in sporting activities and cultural level;

- social safety, including murders and unemployment level of the region;

- social activity, that is participation in elections, meetings, demonstrations and strikes, and membership in informal organisations, in percents of population;

- family structure, i.e. incomplete families and incidence of divorce. 
The indicators have been aggregated aspect-wise according to the formula:

$$
S_{i}=\frac{1}{n_{i}} \sum_{j} \bar{x}_{i j}, \quad r_{4}=\frac{1}{N} \sum_{i} S_{i},
$$

where $S_{i}$ is the index of aspect $i$ (e.g. family structure), $\bar{x}_{i j}$ is the indicator $j$ of aspect $i$ (e.g. incomplete families), $n_{i}$ is the number of indicators in $i$-th group, and $N$ is the number of indicators making up the index.

The state of innovative level have been aggregated into 3-dimensional vector of variables $\theta_{i}, i=1,2,3$ which are assumed to refer primarily to matrices $A, C$ and $A^{z}$ correspondingly as most important because they form the regional efficiency criterion $\kappa(3)$.

After these definitions are made the model coefficients for the region were specified empirically. For the original submodel it was done according the methodology developed in (Gurman, 1987) and described in detail in (Gurman, 2000).

As to the innovative part then corresponding methodology is being developing now based on the following considerstions. It is seen that the empirical parameters are those which connect the innovative activity results with their costs or those which connect the investments with their innovative effects. Inside the economical system there is an important indicator of the effectivity of investment projects which is the interest rate $\nu$. We will try to use it to estimate the parameters under consideration. We assume that the investments are used optimally in the sence of above optimality criteria (6). Its value $\Pi=J_{T \max }$ depends on values of the state variables $k, r, k^{z}$, $\theta, k^{d}$, considered as initial ones whwn calculating $\Pi$. The result of investments can be estimated as an increment of $\Pi, \Delta \Pi$, caused by the increments of those variables which we can take sufficiently small to represent the corresponding dependence as linear. Then

$$
\Pi_{k} \Delta k+\Pi_{k^{z}} \Delta k^{z}+\Pi_{\theta} \Delta \theta=(1+\nu) i^{\text {sum }},
$$

where $\Pi_{k}, \Pi_{k^{z}}, \Pi_{\theta}$ are derivative vectors, and inv $_{\text {sum }}$ is total invest- 
ment in the region.

We use the model equations to express the variables' increments taking them as caused by high intensive investments on small time intervals so that the other terms can be neglected:

$\Pi_{k} u+J_{k^{z}} w-\Pi_{\theta}\left([d]+\left[H_{\mathrm{inv}} u\right]+\left[H_{\mathrm{inv}}^{z} w\right](\theta--\bar{\theta})=(1+r)\left(e u+e w+Q^{d} d\right)\right.$

where $e=(11 \ldots)$ is the identical row-vector to sum up vector components. $[\mathrm{x}]$ denote the "diagonalized" vector $\mathrm{x}$. This equality should hold for any $u, w$, and $d$ which implies a series of concrete relations for unknown diagonal matrices $H_{\mathrm{inv}}$, and $H_{\mathrm{inv}}^{z}$.

As a result of analysis of the situation in Pereslavl region the necessary specifications for the model was made.

$$
\begin{aligned}
& \Gamma(k)=\gamma k, \Gamma^{z}=\gamma^{z} k^{z}, \Gamma^{d}=\gamma^{d} k^{d}, \\
& \Psi^{s}: r_{\min }^{s} \leq r \leq r_{\max }^{s}, \\
& \Psi^{h}: r_{\min }^{h} \leq r \leq r_{\max }^{h}, \\
& W=\sum_{i} w_{i}\left|r_{i}\right|, \text { if } r \notin \Psi^{s}, \quad W=0, \text { if } r \in \Psi^{s}, \\
& \mathbf{\Omega}: \mathbf{L}_{\min }^{\mathrm{dem}} \leq \mathbf{L}^{\mathrm{dem}} \leq \mathbf{L}_{\max }^{\mathrm{dem}}, \\
& L^{\mathrm{dem}}=l^{y} y+l^{z} z+l^{d} d, q u_{\max }+q^{z} u_{\max }^{z}+q^{d} u_{\max }^{d}=u_{\max }^{\text {sum }}
\end{aligned}
$$

Here $L^{\mathrm{dem}}$ and $l^{y}, l^{z}, l^{d}$ are the total labor demand and specific demand row-vectors, $L_{\mathrm{min}}^{\mathrm{dem}}$ corresponds to the admissible unemployment, and $L_{\max }^{\mathrm{dem}}$ is the total labor resource awailable. The last relation means distribution of the total investment awailable in the region $i_{\max }^{\text {sum }}$ between different types of activity according the weight coefficient vectors $q, q^{z}$, and $q^{d}$. It is specified quantitavely in scenarios.

Then the general statistics was gathered, and the basic set of data was formed (Table 1).

The following basic scenarious of the region development were considered.

First scenario (I): continuation of trends of past basic period 19951998 up to 2014 without optimization, and without active control for the ecological and social and innovative components $(z=0$ and $d=0)$. 
Table 1.

Pereslavl region: basic data (the state variables' values refer to the end of 1995)

$\rho=0.05, L_{\min }^{\mathrm{dem}}=27, L_{\max }^{\mathrm{dem}}=43$

\begin{tabular}{|l||l|l|l|l|l||l|l|l|l|}
\hline & 1 & 2 & 3 & 4 & & 1 & 2 & 3 & 4 \\
\hline$k$ & 211 & 251 & 37.3 & & $C_{\text {diag }}^{z}$ & 1 & -1 & 1 & 1 \\
$k^{z}$ & 4 & 12 & 0.15 & 8.5 & $A$ & 0.01 & 0.01 & 0.01 & \\
$k^{d}$ & 10 & 10 & 8 & & & 0.5 & 0.4 & 0.35 & \\
$r$ & 5800 & 8.2 & 69.5 & 0.44 & & 0.001 & 0.006 & 0.06 & \\
$r_{\max }^{h}$ & - & 10 & 200 & 1 & $B$ & 0 & 0 & 0 & \\
$r_{\max }^{s}$ & - & 8.2 & 120 & - & & 0.45 & 0.15 & 0.4 & \\
$r_{\min }^{s}$ & 5000 & - & 60 & 0.4 & & 0 & 0 & 0 & \\
$r_{\min }^{h}$ & 4000 & 0 & 50 & 0 & $A^{z}$ & 0.2 & 27 & 3 & 200 \\
$r^{*}$ & 6000 & 1.2 & 100 & 0.5 & & 0.4 & 33 & 40 & 1000 \\
$\theta$ & 0 & 0 & 0 & & & 0 & 0.2 & 20 & 3000 \\
$\bar{\theta}$ & -0.5 & -0.8 & -0.7 & & $B^{z}$ & 0 & 0 & 0 & 0 \\
$\gamma$ & 0.4 & 0.35 & 0.5 & & & 0.3 & 0.35 & 0.2 & 0.15 \\
$\gamma^{z}$ & 3.7 & 0.019 & 0.03 & 0.0026 & & 0 & 0 & 0 & 0 \\
$\gamma^{d}$ & 0.002 & 0.003 & 0.003 & & $B^{d}$ & 0 & 0 & 0 & \\
$i^{r}$ & 0 & 0 & 0.6 & 0.08 & & 0.3 & 0.3 & 0.4 & \\
ex & 0 & 0 & 0.1 & 07 & & 0 & 0 & 0 & \\
$p_{\text {row }}^{r}$ & 1 & 1 & 1 & & $Q^{d}$ & 50 & 50 & 50 & \\
$l^{y}$ & 0.035 & 0.09 & 0.13 & & $N$ & -0.003 & -4 & -0.05 & 0.01 \\
$l^{z}$ & 0.03 & 0.3 & 2.5 & 40 & & -0.0001 & -0.9 & 0.01 & -0.005 \\
$l^{d}$ & 10 & 10 & 10 & & & 0 & -0.0015 & -0.005 & -0.2 \\
$w$ & 0.6 & 60 & 60 & 4200 & & $10-6$ & -0.005 & -0.0001 & -0.002 \\
$\delta_{\text {diag }}$ & 0.06 & 0.06 & 0.07 & & $C$ & 0 & 0.011 & 0 & \\
$\delta_{\text {diag }}^{z}$ & 0.07 & 0.09 & 0.06 & 0.11 & & -0.0053 & -0.0059 & -0.0034 & \\
$\delta_{\text {diag }}^{d}$ & 0.06 & 0.06 & 0.06 & & & 0.0001 & 0.0005 & 0.0003 & \\
& & & & & & 0.0002 & 0.0001 & 0.0003 & \\
\hline
\end{tabular}


Other scenarios were considered on the period 1999-2014 (15 years). They include approximately global optimization accompanied by iterative improvement.

Second scenario (II): the same as (I) but with approximate optimization without active innovative control, to reveal the level of structural efficiency.

Third scenario (III): the innovative control is activated under different restrictions on investments (several versions). The fastest gaining of structural efficiency is taken for the innovative control program as initial approximation.

Fourth scenario (IY): accounting for the dependence of model parameters on $r$ including positive dependence of innovative matrices on culture and education. Optimization is performed by the universal improvement algorithm.

Fifth scenario (Y): estimation of effect of price change due to changing the regional competability.

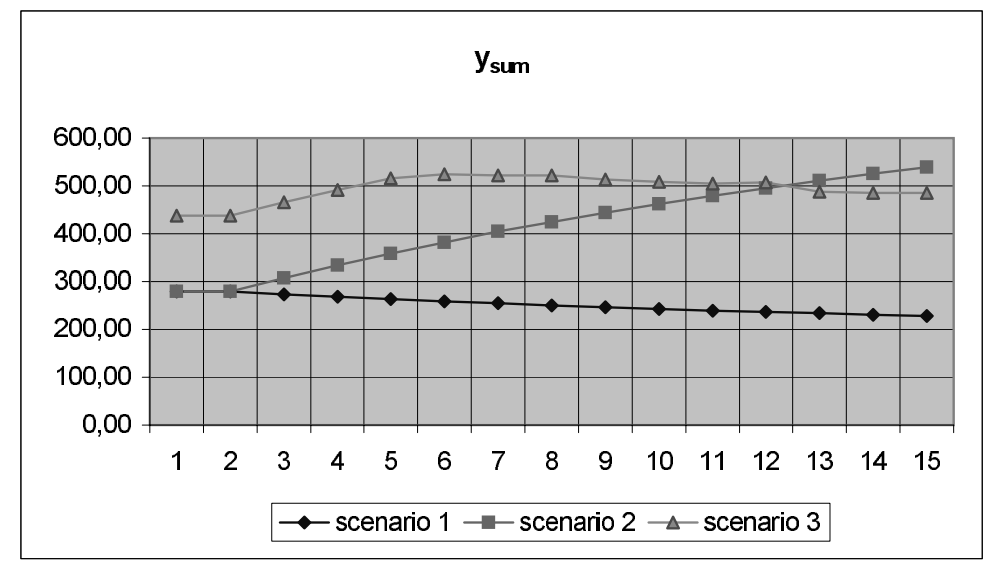

FIGURE 1

The results of model calculations for the first three scenarios are shown on Figures $1-7$ (others are under investigation now). The initial conditions for them were obtained via calculations according 
scenario (I) for 1995-1998. In the whole they are confirmed by preliminary statistical data for 1998, however, it is insufficient to recalculate strictly into the required set of data.

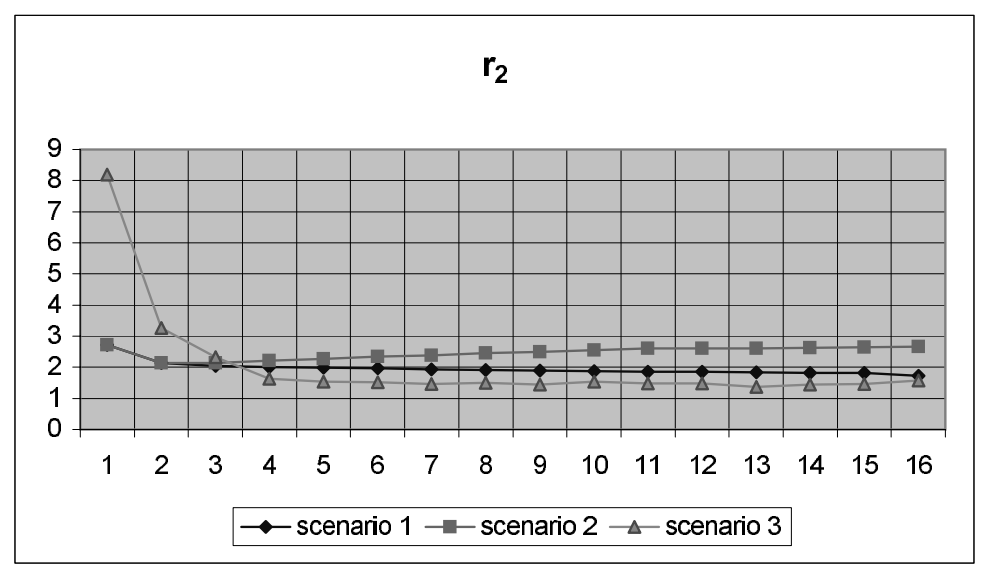

FIGURE 2

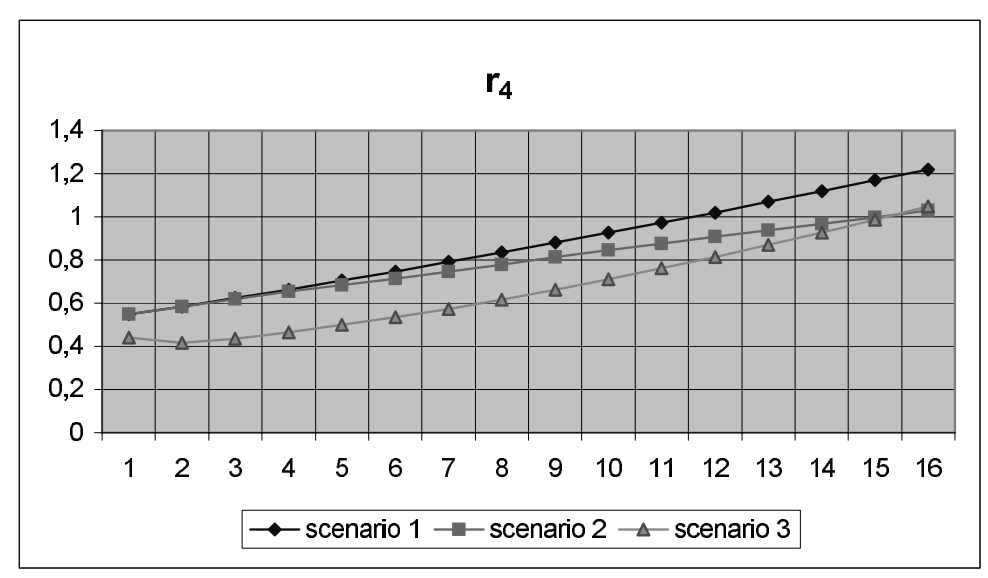

FIGURE 3 


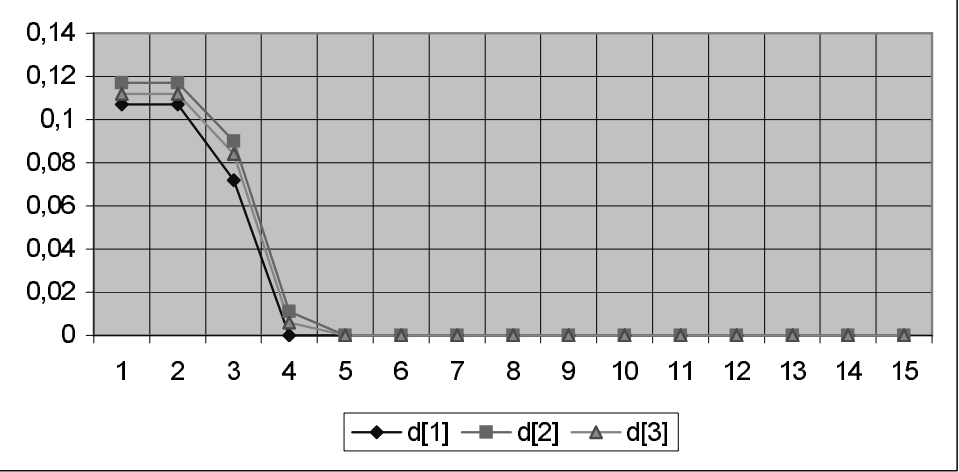

FIGURE 4

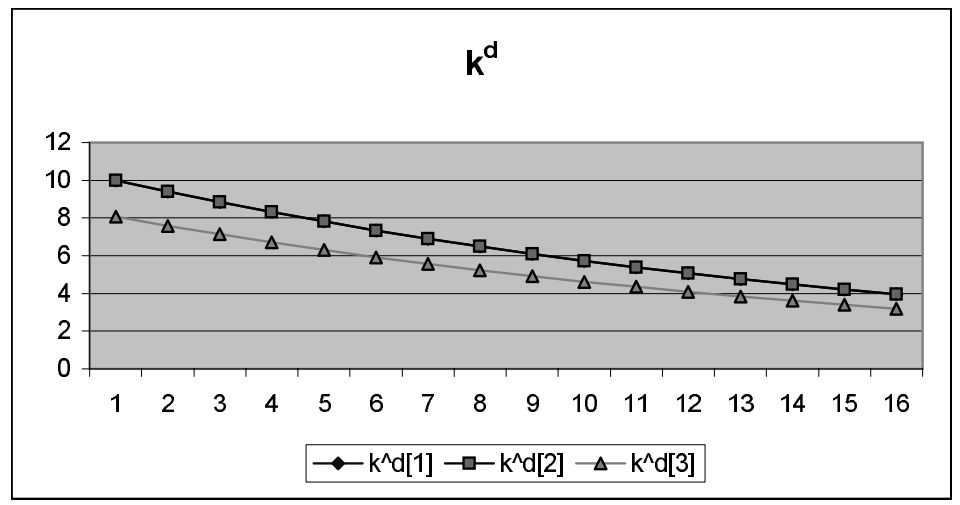

FIGURE 5 


\section{theta}

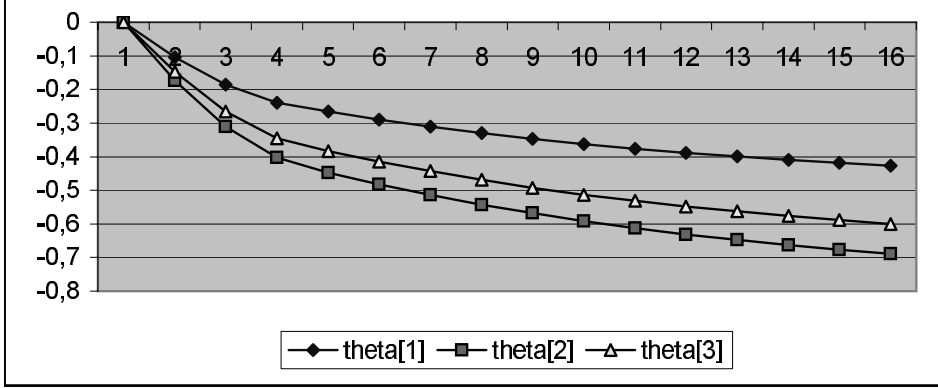

FIGURE 6

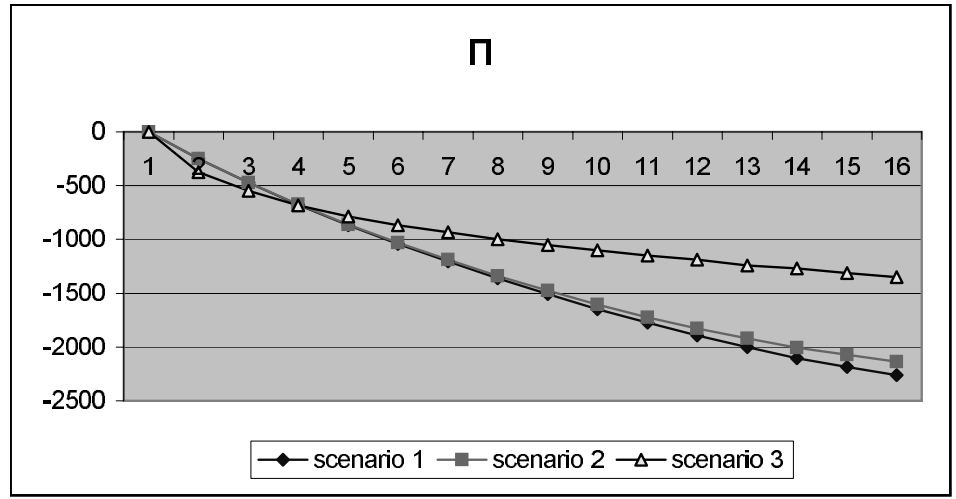

FIGURE 7

As is seen from results for scenario 2, only second aggregated branch of economy is productive and works at the full capacity and with maximal investment in the optimal mode; two others branches are nonproductive; they should work at minimal admissible (from employment considerations) level and without investment. This means that at present time the regional system is structurally ineffective which is also seen from the criterion $(3): \kappa_{1,3}<0$. In the scenario 1 (nonoptimal) this fact is ignored which leds to excessive economic losses (the regional income $\Pi$ in this case is equal to $-2260,561$, much more less its value in the scenario $2, \Pi=-1350$ ), accompanied also by violation of sutainability conditions (3). 
To overcome this drawback in acceptable time horizon active innovative policy is required. As is represented by scenario (III) it consist of radical redistribution the investment to come in favor of the innovative sector which functioning is aimed first of all on improvement the structural efficiency parameters, that is elemets of matrices $A$, $C, A^{z}$.

As a result after 5 years all three aggregated economy branches become productive, that is structural efficiency is attained. After that the investments are redistributed gradually in favor of economic sector and capacities supporting the improvement of most negative ecological and social characteristics

The practical measures to change above unfavorable trends, should include structural reconstructions and diversification in economy, reduction of costs, implementation of resources saving and environment protective technologies, and intensive research and education activities. It is necessary to apply great efforts and resources to improve general image of the region, in all its aspects: infrastructure, ecology, esthetics, and culture, otherwise the available potential of development can not be activated.

\section{Conclusion}

Thus, it is proposed an approach for modelling regional development accounting for innovative processes via adding a special innovative submodel to preceeding socio-ecology-economic model of a region which output results in improving parameters of the original model from the point of view of the accepted criterion, the accumulated discounted regional income under sustainbility restrictions.

The empirical research on the base of this model and succeding computer experiments for a concrete region confirm that it is expedient to use and develop the approach proposed to represent the innovative processes in models of regional development.

The integrated model proposed can be also considered as an in- 
strument for the complex estimation of innovative programs and projects accounting for economy restructuring, and ecological and social efficiency, in contrary with current practice of estimation only financial results of projects.

\section{References}

Belyshev D.V,and Shevchuk E.V.(1999). The algorithm and software packadge for optimal control search, in "Intelligent control", Proceedings of the International Conference, Pereslavl-Zalessky, December 6-9, Nauka • Fizmatlit, Moscow, pp 146-150 (in Russian)

Chow, G.C. (1985).Econometric Analysis by Control Methods Wiley, New York.

Gurman, V.I. (1985, 1997). The extension principle in control problems, Nauka, Moscow (in Russian)

Gurman, V.I, Dykhta, V.A., et al.(1987).Ecology-economic systems: Models, information, and experimentation, Nauka, Novosibirsk (in Russian)

Gurman, V.I.(Ed.)(1990). Ecological-Economic Strategies for Regional Development, Nauka, Novosibirsk (in Russian)

Gurman V.I.,red.(2000).Modeling of a region as a socio-ecologyeconomic system, Nauka, Moscow.

HDR (1995) Human Development Report. United Nations Development Program.

Konstantinov G.N. (1999). Knowledge in the innovative economy. In

"Program systems", Nauka • Fizmatlit, Moscow, pp 262-268 (in Russian). 
Sahal D.(1981). Patterns of technological innovation, AddisonWesley Publishing Company, Inc., Reading, Massachusets, USA. London. Amsterdam. Don Mills, Ontario. Sidney. Tokyo.

Acknowledgements:

The research presented has been carried out thanks to the TACIS ACE Project P-95-4097-R "Design of Regional Ecology-Economic Models for Sustainable Development for NIS countries". We have a pleasure to express our appreciation to its coordinator Christophe Deissenberg for his great help in the research and in preparation of this paper. The optimization part of it has been also supported financially by RFBR, Project 00-01-00731.

We thank Dmitry Belyshev and Elena Shevchuk for their great help in computer experiments over innovative scenarios of Pereslavl region development. 